\title{
DEL ASENTIMIENTO AL CONSENTIMIENTO INFORMADO. FUNDAMENTOS ONTOLÓGICOS Y AXIOLÓGICOS
}

\author{
FROM INFORMED ASSENT TO INFORMED CONSENT: ONTOLOGICAL, \\ EPISTEMOLOGICAL AND AXIOLOGICAL FOUNDATIONS
}

\author{
LUDWIG SCHMIDT H. \\ Universidad Católica Andrés Bello \\ 1schmidt01@gmail.com
}

El poder de la palabra en relación con los asuntos del alma está en la misma relación del poder de los medicamentos en relación con los asuntos del cuerpo

Gorgias de Leontini (s. IV a. C.)

\author{
RECIBIDO: 02/08/2016 \\ ACEPTADO: 02/09/2016
}

\begin{abstract}
Resumen: El asentimiento y el consentimiento informado médico son dos procesos e instrumentos ético-jurídicos del acto-médico, que permiten establecer empatía, comunicación, información, responsabilidad y reciprocidad en el cuidado, consejo y seguridad en la relación médico-paciente, con la familia, la institución prestadora de servicios y los patrocinadores. Constituyen medios de información aptos y adecuados al paciente, sobre la naturaleza de la enfermedad y del procedimiento diagnóstico o terapéutico que se propone utilizar, alternativas, riesgos y beneficios que éste conlleva. El asentimiento y consentimiento forman parte del proceso de relación médico-paciente y constituyen, al final, un documento de acuerdo y contractual, en forma escrita, sobre las responsabilidades mutuas. Pero se diferencian, en que el primero pertenece más a la voluntad y el segundo, representa un acto del entendimiento. Se han venido aplicando en la relación de niños y adolescentes, con enfermos crónicos y sus familiares-cuidadores u otros, con enfermedades neurológicas que pierdan momentáneamente su competencia mental.
\end{abstract}

Palabras clave: acto médico, asentimiento informado, consentimiento informado, voluntad, reciprocidad, proceso y documento de acuerdo curativo.

\begin{abstract}
The Informed Assent and Informed Consent are two ethical and legal processes instruments of the act-doctor, which establish empathy, communication, information, responsibility and reciprocity in health care, advice and safety in the doctor-patient relationship with the family, servicing institution and sponsors. Media are suitable and appropriate to the patient, the nature of the disease and the diagnostic or therapeutic procedure proposed use, alternatives, risks and benefits it brings. Assent and consent form part of the doctor-patient relationship and are ultimately a contractual agreement document and in written form on the mutual responsibilities. But they differ in that the first belongs more to the will and the second, represents an act of understanding. They have been applied in the relationship of children and adolescents with chronically ill and their relativescaregivers or others with neurological diseases that momentarily lose their mental competence.

Key words: medical act, informed assent, informed consent, will, reciprocity, healing process and document agreement.
\end{abstract}




\section{Introducción}

El presente artículo, inscrito en la línea de investigación Fundamentos de Bioética Clínica, trata de dos protocolos de Buenas Prácticas (B.P.): El asentimiento informado (A.I.) y el consentimiento informado (C.I). Éstos, se circunscriben en el acto médico corresponsable, centrado en la: (a) comunicación médica eficaz; (b) información apta, adecuada y oportuna; y, (c) participación del paciente en la decisión de su propio cuidado y afrontamiento del porvenir. Protocolos formales de Autonomía y Justicia en la Medicina Participativa del siglo XXI con fundamento ancestral en la Medicina.

El clásico aforismo de Gorgias enunciado en el epígrafe, da luces sobre la importancia del valor de la palabra en su relación con otros congéneres, más aun cuando ésta, pueda ser dispar (médico-paciente). Luego, si el acto médico es más que el mero hecho de curar y prolongar la vida ${ }^{1}$, dando instrucciones de manera paternalista en la procura de la beneficencia (principia prima facie). La historia ha impuesto el cambio de paradigma de atención médica, al docenteparticipativo, donde el médico enseña-al-otro a: (a) aprender-a-conocer: capacitándolo sobre el tratamiento, concienciándolo con un consejo adecuado y oportuno, sensibilizándolo sobre el valor de la salud y, responsabilizándolo del acto curativo; (b) aprender-a-aprender la toma autónoma de decisiones y desarrollar un pensamiento crítico (Si bien para el A.I. no aplica, se informa); (c) aprender-a-hacer participar al paciente en el rol del cuidado médico, como cuestión relativa a la sostenibilidad de su salud; (d) aprender-a-ser, valorando su vida, dignidad, autonomía ${ }^{2}$ de voluntad e intimidad, tras recibir información adecuada que fomente el descubrimiento y la experimentación, y por escrito, para desarrollar su criterio y personalidad. El valor de la palabra enseña a padres e hijos, representantes y representados la importancia de ambos procesos e instrumentos (A.I. y C.I.).

Teniendo en cuenta que el A.I. y el C.I son parte del acto médico, cada uno de ellos tienen fines diferentes (Acceso a la información y Autonomía, respectivamente), a su vez, son complementarios e integradores de la dimensión biológica, psicológica y ecosocial de las personas (médico y paciente, de la

\footnotetext{
${ }^{1}$ Cf. Callahan, D: The goals of medicine: Setting new priorities. NY: The Hasting Center Report; 1996.

2 La autonomía del paciente como bien jurídicamente protegido surge como una manifestación de la libertad humana y del reconocimiento de su dignidad y valor de la persona, tal y como ha sido plasmada en la Declaración Universal de los Derechos Humanos.
} 
Institución y familia, de patrocinadores y la sociedad en general) ${ }^{3}$. Por lo tanto, el tema-eje en este artículo estará en la importancia del saber-comunicar e informar-eficazmente ${ }^{4}$ el acto médico a su contraparte que es "competente" para saber o no-saber, de ser capaz de entender lo-que-se-le-dice; de poder aceptar o no-aceptar "algo" que se le prescriba. Todo lo anterior, enmarcado en la Relación Médico-Paciente (R.M.P.) en una Unidad de Prestación de Servicios de Salud (U.P.S.S.) frente a su familia y su contexto de vida.

La R.M.P. es compleja y debe ser colaborativa por las partes, para aprendera-sanar, que el ir al médico no es un castigo, sino una necesidad que es a su vez grata, sobre todo a los menores, personas que sufren alguna deficiencia ${ }^{5}$, discapacidad $^{6}$ o minusvalía ${ }^{7}$, los que están en prisión o pacientes psiquiátricos, permitiéndoles un ambiente más tolerante y cómodo ante las adversidades y capacidades críticas de la vida. Por todo lo anterior, el médico debe repensar sus estrategias de abordaje de la asistencia e investigación médica en estos tiempos postmodernos y posthumanistas, a saber, desde los puntos de vista: (a) Ontológico $^{8}$, el médico se observarse a sí mismo frente al acto médico como constructor y reconstructor del ámbito clínico, al ubicarse en una realidad sociocultural como proceso histórico, en donde su acción médica y educativa, continuamente produce y reproduce innovaciones el mundo material, social y cultural en el cual vive; (b) Epistemológico ${ }^{9}$, el médico establece las condiciones

3 Schmidt, L: El ser humano: visto desde el paradigma holístico. Saarbrücken: Editorial Académica Española, 2013.

${ }^{4}$ La información debe ser verdadera, y debe comunicarse al paciente de forma comprensible y adecuada a sus necesidades, con el objeto de que éste pueda tomar las decisiones referentes a su salud de acuerdo con su libre y propia voluntad.

5 Deficiencia se refiere a toda pérdida o anormalidad de una estructura o función psicológica, fisiológica o anatómica. Por ejemplo, la pérdida de la vista, la pérdida de la memoria o la pérdida de un miembro [O.M.S.].

${ }^{6}$ Discapacidad se refiere a toda restricción o ausencia de la capacidad de realizar una actividad en la forma o dentro del margen que se considera normal para un ser humano. Por ejemplo, la perturbación en la capacidad de aprender, o la dificultad para vestirse por sí mismo [O.M.S.].

7 Minusvalía se refiere una situación desventajosa para un individuo a consecuencia de una deficiencia o discapacidad que limita o impide el desempeño de un rol que es normal en su caso en función de la edad, sexo o factores sociales y culturales. Por ejemplo, la imposibilidad de conseguir empleo, la ausencia de relaciones sociales o los estados depresivos y de ansiedad frecuentes [O.M.S.].

${ }^{8}$ Pudiera decirse que es el estudio de lo que es en tanto que es. Por ello es llamada la teoría del ser, es decir, el estudio de todo lo que es: qué es, cómo es y cómo es posible. La Ontología se ocupa de establecer las categorías fundamentales o modos generales de ser de las cosas.

${ }^{9}$ Pudiera decirse que corresponde a un objeto de estudio puede enfocarse bajo diferentes paradigmas que permiten conducir a diversas observaciones que dependen del marco conceptual y metodológico 
básicas que dan soporte a la coherencia y validez del acto médico en un mundo postmoderno, por cuanto atiende a las necesidades del proceso investigativo, sin olvidar que su paciente es un ser-humano y no un mero objeto de estudio. Además, desde el punto de vista filosófico y metodológico, le sirve de base (A.I. y C.I.) y justifica los procesos, para la autónoma toma de decisión del paciente menor-adulto o con alguna deficiencia, discapacidad o minusvalía y que no-tiene o ha perdido parcialmente su competencia mental y legal e intenta dar solución a un problema de clínico. Por lo tanto, hay que tener claro el trasfondo ontoepistemológico de las B.P. de asistencia y de investigación médica; y (c) Axiológico $^{10}$, el médico aplica esta estructura médica (tecnocientífica y bioética) a un concepto y poder ordenar y jerarquizar sus propiedades, partiendo de la base que el sujeto de la valoración es algo extrínseco y ocupa un espacio en un tiempo determinado. De allí, la identificación de su dimensión sistémica, extrínseca e intrínseca y posteriormente, las combinaciones que puedan realizarse en la medida en que se profundiza en el conocimiento del objeto de valoración.

La R.M.P. está sujeta a otras reglas éticas y epistemológicas que le hacen posible a modo de conditio sine qua non el acto médico (docente, asistencial e investigativo), en las B.P. específicas. A su vez, con una exigencia adicional, el "ser bilingüe". En otras palabras, al requerir de un lenguaje comunicativo, con medios pedagógicos y didácticos que faciliten el entendimiento y la comprensión decisoria del paciente ${ }^{11}$. La comprensión común de los enunciados que se argumenten en pro de la vida, la salud y la ciencia, corresponden a un pensamiento específicamente médico-filosófico $\mathrm{y}$, presentes en general, en cualquier acuerdo intersubjetivo que se establezca entre las dos partes de la

a través del cual pueden observarse. Al conjugar la teoría y la aplicación de la ciencia, es un requerimiento el uso de la epistemología para poder interpretar y conocer una realidad. Corresponde a la teoría del conocimiento científico y ésta, se caracteriza por su método. Razón por la cual la epistemología es la doctrina de los fundamentos que presenta el conocimiento como el producto de la interacción del hombre con su medio e implica un proceso crítico a través del cual el hombre ordena el saber hasta llegar a su sistematización.

${ }^{10}$ En el caso concreto la investigación, la estructura axiológica se refiere a la protección, la seguridad y la curación. Su ámbito de acción al identificar, jerarquizar y valorar las necesidades del sistema, intrínsecas y extrínsecas, propias de su actuación en el quehacer médico. Por ello, el enfoque epistemológico que la fundamenta es el interpretativo-crítico signado por unas referencias de validación situadas en los simbolismos socio-culturales de un momento témpora-espacial y apoyado en una concepción humanístico-científica. Si el axioma fundamental de la axiología formal, habla de la relación de bondad de un objeto, con el mayor o menor grado de cumplimiento con las propiedades del concepto de ese objeto, ella permite ordenar y ponderar las propiedades de un concepto, al utilizar las mismas dimensiones axiológicas, para lograr una mejor valoración.

${ }^{11}$ Ello se hace con el propósito de ser corresponsable de la curación y afrontamiento de éste, en su ecoambiente en un porvenir. 
R.M.P., así como del dictamen médico (preventivo, diagnóstico o terapéutico) requieren en de "experticia" y de un "diálogo" ético eficaz y fraterno. Tal y como diría Karl-Otto Apel, el lenguaje implica a los hablantes desde el mismo momento en que éstos, se atribuyen mutuamente una relación pragmática, en cuanto sujetos que intentan ponerse de acuerdo. Naturalmente, esta pragmática lleva a una ética de la comunicación o ética del discurso, que comparte con Jürgen Habermas, desde el momento que, en última instancia, toda posibilidad de comunicación exige, no sólo una normativa, sino también la actitud moral de aceptar al otro como persona ${ }^{12}$ y su autonomía ${ }^{13}$.

\section{En torno a la verdad}

El tema de la comunicación, de la información y de la toma de decisiones son en esencia temas antropológicos y éticos fundamentales, propios de la ciencia en general, que busca el conocimiento y el mayor bien al ser humano, los que conjuntamente, se denomina el bien-ser del hombre ${ }^{14}$, cuyo fin último y supremo

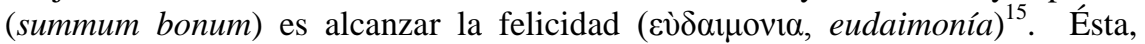
consiste en la actividad del espíritu humano por el conocimiento de la verdad, actividad que es conforme a su naturaleza y responde a su propia teleología. La búsqueda de la verdad ${ }^{16}$ es connatural del ser humano y constituye una virtud

${ }^{12}$ Appel, KO: Teoría de la verdad y ética del discurso. Barcelona: Paidós, 1991.

13 En su formulación y estudio ha admitido variantes doctrinales, siendo Kant y Max Scheler los filósofos más representativos a este respecto. Sin embargo, no podemos omitir una primera formulación anterior a Kant, dada por el teólogo Vázquez, y otras muy significativas de Messer y N. Hartmann.

${ }^{14}$ Cf. Schmidt, L: El hombre como ser ecosocial. En Revista de Bioética Latinoamericana, En Revista de Bioética Latinoamericana. Mérida: SABER-Universidad de Los Andes vol. 8(2) 2011: 1835. Disponible en http://www.saber.ula.ve/bitstream/123456789/34037/1/ articulo3.pdf ISSN: 22447482 Ibídem: El ser-trascendente: Una perspectiva judeocristiana. En Revista de Bioética Latinoamericana. En Revista de Bioética Latinoamericana. Mérida: SABER-Universidad de Los Andes Vol. $10 \quad \mathrm{n}^{\mathrm{o}}$ 1: Septiembre - Febrero 2012: 53-99. Disponible en http://www.saber.ula.ve/bitstream/ 123456789/36074/1/articulo4.pdf.pdf. También en http zs://www.yumpu.com/es/document/ view/13101286/ver-pdf-saber-ula

${ }^{15}$ Originariamente, doctrina filosófica griega que puede atribuirse sobre todo a Sócrates, Platón y Aristóteles, aunque desarrollada sistemáticamente sólo por este último en la Grecia Antigua, lo constituye: Aristóteles: Ética a Nicómaco, 1.1, 4, 1095 ${ }^{\mathrm{a}}$. Madrid: Centro de Estudios Constitucionales, 1985, p. 3.

${ }^{16}$ Cf. Schmidt, L: Fe, ciencia y bioética. En rev. latinoam. bioet., Dic 2011, vol.11, no.2, p.22-41. Disponible también en http://www.umng.edu.co/documents/10162/805711/Bioetica21-pags22- 
que se alcanza con el conocimiento y la vivencia. Corresponde al ejercicio propio de la vocatio-medica ancestral y propia del acto médico per-se, mediante la B.P. y la satisfacción de sus pacientes.

La verdad, insumo del bien-ser, es un concepto polisémico (del latín veritas) y transdisciplinar. Su etimología occidental, proviene: (1) del hebreo (la voz 'emeth, cuyo radical 'aman significa sostener algo de forma firme, para que no caiga. Entre los sinónimos que acompañan o sustituyen a 'emeth, el principal es 'emunah, fidelidad); (2) del griego (la voz $\dot{\alpha} \lambda \eta \dot{\theta} \theta \varepsilon 1 \alpha$, alétheia, compuesto de negación y la raíz del verbo lanthano, estar oculto; por tanto se refiere a «lo-queestá-patente») ${ }^{17}$. Su conceptualización se ha ido sobreponiendo "paramétricamente" durante el transcurso del tiempo, a saber: (1) del hebreo, y demás pueblos semíticos, la verdad representa una concepción teológica, de algo-dado al ser (amor), revelado por la divinidad para la vida ${ }^{18}$. Por ello, la verdad era Absoluta, por ser revelada por Dios a los hombres, teniendo un carácter más dinámico según los "signos de los tiempos" y porque "así-es" (amén); (2) en un principio, en la Grecia Antigua, la preocupación por la verdad era más ontológica y estuvo inicialmente ligada a la búsqueda del arché, término expresa la razón de ser, el fundamento, la verdad más profunda de las cosas; (3) con Sócrates, Platón y Aristóteles, se comienza a emplear el concepto griego alétheia, algo más epistemológico. La verdad representó lo-que-es o lo-que-no$e s$, que se remite a lo permanente, a lo que tengo "que quitar el velo", para conocerlo. De allí, el método socrático denominado: "mayéutica" (gr. $\mu \alpha \iota \varepsilon v \tau ו \kappa \eta)$ que designa el arte de la comadrona o partera ${ }^{19}$; estableciéndose así la "conformidad que existe entre lo que se dice, piensa o cree y la realidad", lo-quees o lo-que-sucede ${ }^{20}$. Se le comenzó a definir aspectos teleológicos y axiológicos; (4) con la Escolástica medieval, Tomás de Aquino ${ }^{21}$ se define como

41.pdf; y en http://www.scielo.org.co/scielo.php?script=sci_arttext\&pid=S1657-47022011000200 004

${ }^{17}$ Wiki de la Encyclopaedia Herder. Brugger: Diccionario de Filosofía. Barcelona: Herder, 2016.

18 Schmidt, L: La Buena Nueva y la Bioética. Disertación entre la vida y la ciencia desde el relato bíblico del génesis. Caracas, San Pablo: Colección Biodiké No 4, 2008. 64 pp.

${ }^{19}$ Sócrates ejerce una mayéutica mental, ya que en sus diálogos interroga a sus interlocutores, para ayudarles a alumbrar ideas, que tampoco él ha engendrado, sino que estaban ya en la mente de sus interlocutores a pesar de que éstos no lo supieran. En ambos casos de trata de ayudar a alumbrar un producto que ni la comadrona ni el maestro (Sócrates) han engendrado.

${ }^{20}$ Esta concepción fue la que más se difundió en Europa, Norte del África y Medio oriente Así se ha entendido tradicionalmente la verdad, interpretada como correspondencia, o coincidencia, entre la mente y la realidad o los enunciados y los hechos. En sentido estricto es la correspondencia de una proposición o enunciado con los hechos.

${ }^{21}$ Tomás de Aquino, Quaestio Disputata De Veritate. Qu.1 
la «adecuación entre el entendimiento y las cosas», hasta los lógicos modernos, entre ellos. En líneas generales, la Edad Media mantiene las tesis de los Antiguos, §(3), con algunas modificaciones procedentes del papel conferido a la divinidad. Así, en Agustín de Hipona, cabe destacar su obra Contra academicos, el estatuto del filosofar como búsqueda de la verdad. La Verdad está en (o es) Dios, pero el hombre encuentra verdades parciales ${ }^{22}$; (5) Alfred Tarski, aceptó este último concepto de verdad $\S(4)$, como correspondencia y lo ha liberado de todas las connotaciones metafísicas, construyendo la denominada teoría semántica de la verdad; (6) por otro lado, no todos los enunciados verdaderos lo son por su correspondencia con los hechos. La teoría de la adecuación o correspondencia debe complementarse con la de la coherencia, y con la teoría pragmática de la verdad.

Para ser verdadera, una experiencia perceptual o conceptual debe conformarse con la realidad [...].Por «conformidad» el humanismo [pragmatismo] entiende tomar en cuenta de tal modo que se obtenga algún resultado satisfactorio intelectual y prácticamente. «Tomar en cuenta» $\mathrm{y}$ «ser satisfactorio» son términos que no admiten definición, dado que son tantos los modos en que estos requerimientos pueden desarrollarse prácticamente ${ }^{23}$.

Desde el punto de vista bioético en el acto médico, la verdad no es absoluta sino relativa, basada en una ética de los mínimos y de los derechos humanos fundamentales. Por otro lado, no se habla de verdad relativa, sino se le refiere al sentido de "veracidad" o de "amor a la verdad", lo que significa no sólo la coincidencia del hablar con la persuasión interna (en contraposición a la mentira), sino también la coincidencia de la acción externa con la actitud interna (en contraposición a la ficción), así como el enjuiciamiento honrado de sí-mismo (en contraposición al autoengaño) y la voluntad leal de llegar al conocimiento de la verdad.

El médico dentro de la tradición hipocrática, toma de Sócrates la estrategia de interrogar a sus interlocutores, de pedir evaluaciones de soporte, las cuales examina junto con aquello que el alma del interrogado ha producido, lo que él ha observado, y reflexiona con base en sus conocimientos y consultas. El proceso mejora cuando el interlocutor descubre la verdad (las causas y consecuencias) a partir de una serie bien trabada de preguntas y respuestas, y del examen de las

22 En cuanto que son verdades, participa de la divinidad, al tiempo que cumple el objetivo de la filosofía.

${ }^{23}$ James, W: El significado de la verdad. Madrid: Aguilar, 1980, 5 ed., p. 116. 
inconsecuencias que las respuestas originan. En la medida en que, en el proceso del diálogo, el interlocutor permite al médico entender las causas y las consecuencias que generaron la enfermedad y el paciente aceptar las incongruencias que revelan sus respuestas, el proceso de curación se inicia. La mayéutica socrática está en línea con la teoría platónica de la anamnesis o reminiscencia ${ }^{24}$. No obstante, aunque Platón no se contenta solamente con la mayéutica, sino que su método se basará fundamentalmente en la dialéctica, nunca renunció a la dimensión irónica, interrogativa y aporética de la mayéutica socrática. Este método se va mejorando en el tiempo, adaptándose a las necesidades y expectativas de las personas en sus contextos socioculturales. Así, el apto, adecuado y oportuno proceso conduce al aprender-a-conocer ${ }^{25}$ y al aprender-a-ser ${ }^{26}$ del interlocutor. La vocatio-medica exige además de un compromiso ante el acto médico que se práctica y en su R.M.P. particular. El término proviene del latín com-promittere, obligarse mutuamente a aceptar la decisión ${ }^{27}$ de un tercero que actúa como médico o juez en una cuestión. De esta manera, se logra el aprender-a-hacer ${ }^{28}$ y el aprender-a-vivir ${ }^{29}$. Término que se

\footnotetext{
${ }^{24}$ Platón introduce este concepto en el Menón (80e-81e), y lo sigue mencionando en el Fedón(72c y siguientes), en el Fedro (72e-77a, 249b-250a), en La República 604d, y en las Leyes 732a. En el Menón (81b) afirma que investigar y aprender son, en definitiva, anámnesis (ḋvó $\mu v \eta \sigma ı)$ ).En este diálogo entre Sócrates y Menón (discípulo del sofista Gorgias, que había afirmado que «si algo existiese no podría ser conocido, y si algo pudiese ser conocido no podría ser comunicado por medio del lenguaje»), se plantea la cuestión del principio heurístico según el cual conocer o aprender es un proceso imposible a menos que ya se conozca de alguna manera aquello que se busca, puesto que no es posible investigar lo que ya se sabe (si ya se sabe, ¿para qué buscarlo?), ni es tampoco posible investigar lo que no se sabe (si no se sabe qué investigar, ¿cómo investigarlo y cómo saber que lo hemos hallado?)

${ }^{25}$ Consiste en que el consultante, paciente o lesionado en aprenda a comprender y a interpretar el mundo que le rodea, de manera que le permita vivir con dignidad, desarrollando sus capacidades perceptuales y comunicarse con los demás.

${ }^{26}$ Consiste en que el consultante, paciente o lesionado desarrolle el autoconocimiento, por le permita todo un viaje interior, cuyas etapas corresponden a las de la maduración constante de la personalidad y del carácter.

${ }^{27}$ En la etimología de la voz que nos ocupa encontramos, fundamentalmente, dos significados: el de obligación (vínculo que muestra una exigencia a la voluntad) y el de promesa (expresión de la voluntad de actuar de cierto modo). Por un lado, hallamos pues, la referencia al verbo latino ob-ligo (atar o unir con) y por otro, al verbo promitto (prometer o enviar en defensa de). Así llegamos a la síntesis preliminar de lo que pueda designar el concepto compromiso: «Vínculo que presenta una exigencia de respuesta adecuada a la voluntad».

28 Consiste en que cada uno aprenda a hacer, y está vinculado al aprender a conocer, por lo que van unidos y tiene el sentido de poner en práctica los conocimientos que se van adquiriendo.
} 
corresponde con el engagement del existencialismo francés. La actuación didáctica-pedagógica del médico en develar causas y efectos, intensidades, riesgos y complejidades, requieren que la empatía entre médico y paciente se refleje en intencionalidades que comprometan su mejoramiento y la calidad de su investigación.

\section{Asentimiento informado médico}

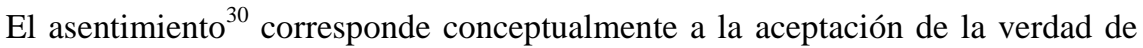
un enunciado o proposición, o de la verdad que se considera que contiene una doctrina o creencia, o conformidad con lo que expresa una norma moral o un mandato jurídico. Su desarrollo etimológico ${ }^{31}$ tuvo lugar con: (1) los escépticos griegos, quienes enseñaban la suspensión o abstención del asentimiento ante la

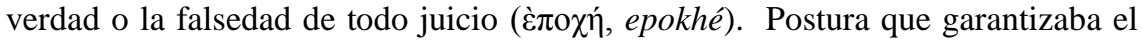
valor de la palabra dada en esos tiempos y que perdurara hasta la actualidad en ciertas comunidades; (2) la filosofía escolástica, el asentimiento es parte del juicio, y se expresa mediante la unión o la cópula, el verbo «ser». De esa manera, se establecía un nexo entre dos personas de manera particular (p. ej: de seguir las instrucciones terapéuticas del médico, entre el hombre y la mujer en matrimonio, de cumplir por parte de un sentenciado el dictamen del juez); (3) los filósofos y psicólogos hablan en la actualidad, el asentimiento se le atribuye un orden propiamente mental; (4) los teólogos, filósofos, políticos y en este caso, los médicos, hablan de un asentimiento moral o real, que no se da a la verdad de un enunciado, sino a una doctrina moral, religiosa o política, que se acepta, y con el que se expresa el compromiso de la persona al respecto ${ }^{32}$. Se asumirá esta acepción terminológica en el artículo.

Uno se pregunta: ¿Cómo se emplea este concepto en medicina? ¿Por qué se le asemeja al C.I.? ¿Qué diferencias existen entre el A.I. y el C.I.? Tal vez, todo sea cuestión de moda o innovaciones. ¿Pero esto es cierto? ¡No! En un ejercicio deductivo: (a) ambos términos están en función de la autonomía, entendida desde

\footnotetext{
${ }^{29}$ Consiste en que cada uno aprenda a relacionarse con los demás, de manera que evite adversidades o conflictos y los solucione, fomentando el conocimiento de los demás. Siendo más tolerantes, inclusivos y por tanto multiculturales e interculturales, para poder vivir y ayudar a vivir.

${ }^{30}$ Se refiere al acto de asentir, lo que significa el admitir como cierto o conveniente lo que otra persona ha afirmado o propuesto antes [DLE].

31 Wiki de la Encyclopaedia Herder. Brugger: Diccionario de Filosofía. Barcelona: Herder, 2016.

32 Cf. Newman, JH: El asentimiento religioso. Ensayo sobre los motivos racionales de la fe. Barcelona: Herder, 2010.
} 
el punto de vista kantiano de la «capacidad de darse a sí mismo normas, con vistas a la praxis, y asumir la propia vida en función de dicha posición». Denunciado anterior, se entiende que no existe nada en este mundo que resulte tan universal como la conciencia libre y autolegiferante, esto es, hacedora de leyes; (b) la asunción de una enfermedad, afección o a participación experimental, genera un compromiso y conductas de autoobediencia, ¿pero siempre es así? Lamentablemente, la experiencia indica que no. Luego, no se puede plantear una autonomía absoluta, por lo que hay que prever márgenes de tolerancia. La autonomía tendrá que asumirse no sólo desde la voluntad sino también desde la autonomía para la acción, es decir, en autogestión. Aunque en ocasiones, esta se ve contradicha en la práctica, cuando la persona delega las decisiones de la propia conciencia en manos de otra persona; (c) el A.I. y el C.I. tienden a la autogestión responsable de su proceso clínico (formativa, diagnóstica, terapéutica e investigativa), por el compromiso que se adquiere con el médico tratante y su familia. El A.I. y el C.I. requieren del "atreverse-asaber" más sobre su participación, proceso, alternativas, riesgos $\mathrm{y}$, para "atreverse-a-decidir y a-elegir", sabiendo que, aunque se equivoque, si la decisión fue tomada en conciencia, se ha hecho sinceramente, que has hecho ética y personalmente lo que se debía hacer; (d) en general, el concepto de asentimiento es, en oposición al consentimiento, algo que pertenece a la voluntad, un acto del entendimiento, de compromiso personal de hacer algo que necesito hacer en procura de mi mayor bien: la salud, en este caso; (e) el A.I. permite el derecho de ser informado pero no sobre consentir la terapia. Mientras que el C.I: es la expresión de voluntades en la formación del contrato mismo, y el A.I. responde a la legitimación para el acto de ejecución, y que el consentimiento-legitimación se relaciona con el ejercicio de un derecho de la personalidad y la integridad física. Entonces, el A.I. es una declaración de voluntad no negocial, que integra un acto voluntario lícito; (f) esta distinción pondría en relieve que el consentimiento contractual es distinto del que se requiere para cada acto que importe la afectación de la libertad del paciente. Ello permitiría a los menores tener capacidad para asentir tratamientos médicos. El A.I. es un acuerdo de responsabilidad para seguir un tratamiento. El C.I. representa un "contrato médico".

El A.I. no es un retrabajo, ni un modelo simplificado del C.I., ni una versión para personas con "incompetencia legal" a comprometerse. Es un proceso complementario, para que quién es representado jurídicamente, se sienta que él también está social y médicamente participando en el mejoramiento de su 
salud $^{33}$. El documento del A.I. es didácticamente diseñado y pedagógicamente estructurado conforme a la edad y a la condición personal y tipo de padecimiento. Éste, generalmente comprende: $\left(1^{\circ}\right)$ Encabezado o portada con el nombre (Actividad o Proyecto, Médico o investigador, Institución y Patrocinador); $\left(2^{\circ}\right)$ Nota destacada de que el A.I. no reemplaza al C.I.; $\left(3^{\circ}\right)$ Breve explicación del procedimiento, indicando objetivos y secuencia de actividades a ser realizadas; y complementos explicativos del proceso con preguntas a ser contestadas en su oportunidad, de forma adecuada; $\left(4^{\circ}\right)$ Cronograma que señala las fechas de las citas para el control de sus resultados, y un seguimiento de la evolución; $\left(5^{\circ}\right)$ Derechos: Confidencialidad de la información, Negarse a continuar; $\left(6^{\circ}\right)$ Beneficios, riesgos y compensaciones que pudieran presentarse, con cuestionamientos de breve desarrollo, selección múltiple o de escala. Estos serían respondidos en momentos particulares; $\left(7^{\circ}\right)$ Acciones de emergencia (situaciones adversas). ¿Qué y cómo hacer? ¿Qué pasó? $\left(8^{\circ}\right)$ Resultados y evaluación, una coevaluación para sus "cuidadores" y autoevaluación que indique el avance del proceso seguido; $\left(9^{\circ}\right) \mathrm{La}$ firma de asentimiento en el inicio y en diversas etapas, para confirmar el logro alcanzado. Éste documento pudiera ser un pequeño cuaderno, una carpeta personal, preferiblemente foliado y manejable para su usuario.

\section{Consentimiento informado}

Pedro Laín Entralgo ${ }^{34}$, expresa que existe C.I. si-y-solo-si cuando: (1) existe una verdadera relación de mutua confianza, establecida en la comunicación eficaz entre médico y paciente; (2) es necesario que el paciente tenga criterios que les permita decidir sobre el tratamiento o las exploraciones a realizar. En otras palabras, sea colaborador en el tratamiento y en las exploraciones; (3) pero para que el paciente pueda decidir es necesario que el médico, considerado como el experto, informe correctamente al paciente de lo que le piensa hacer. Como parte del acto médico, el procedimiento garantiza que el sujeto ha expresado voluntariamente su intención de participar en la investigación (suministrando

33 Experiencias a reproducirse en el mundo, sobre todo en la Pediatría, Oncología Pediátrica, p.ej: Katz AL, Webb SA, Committee on Bioethics: Informed Consent in Decision-Making in Pediatric Practice. En Pediatrics. 2016 Aug; 138(2); Poston RD: Assent Described: Exploring Perspectives From the Inside. En J Pediatr Nurs. 2016 Jul 8; Lepola P, Needham A, Mendum J, Sallabank P, Neubauer D, de Wildt S. Informed consent for paediatric clinical trials in Europe. En Arch Dis Child. 2016 May 25.

34 Cf. Laín Entralgo, P (1983): La relación médico-enfermo. Madrid: Alianza Universidad. Madrid. 
datos e informaciones que garanticen la confidencialidad, ser un integrante de un ensayo o investigación determinada y dentro de un lapso y condiciones específicas) y que después de haber comprendido la información que se le ha dado, acerca de los objetivos del estudio, los beneficios, las molestias, posibles riesgos y alternativas, se establecen sus derechos y responsabilidades de participar o retirarse, de ser atendido en casos adversos, entre otros detalles.

Se cuenta desde los años 50 del siglo pasado, diversos documentos de C.I. que han ido mejorándose durante más de seis décadas ${ }^{35}$. La experiencia ha permitido un amplio desarrollo, aunque en algunos se observan deficiencias de forma y fondo, por intentar replicar el documento de ciertas especialidades en otras, sin saber cómo diferenciarlos.

\section{Epílogo}

A.I. y C.I. es parte del acto médico en la medicina del siglo XXI. Ambos procedimientos permiten el Derecho a ser informado y el C.I. es un documento que protege la Autonomía del Paciente. Este proceso se hace dual, sólo en ciertas circunstancias donde la responsabilidad legal les impide a sus pacientes su autonomía plena en la decisión y se requiere de su compromiso en el seguimiento y control de la actividad clínica e investigativa. La R.M.P. responde a la vocatio-medica deberá estar basada en la comunicación, confianza y el poder del conocimiento médico, que respeta a su paciente y le permite la asistencia clínica y la investigación biomédica ante la necesidad de conocer las situacioneslímites del cuerpo humano y sus enfermedades, de los nuevos productos farmacológicos, instrumentos y prótesis biomédicas, vacunas, terapias quirúrgicas y otros tratamientos. Esta relación desde una perspectiva más participativa y bioética (medicina centrada en el paciente), pretende una autonomía de la conciencia, junto con una autonomía para la acción, es decir, en autogestión. ¿Pero esto será posible en la actualidad? Sobre todo cuando la autonomía reivindica para la moral un estatuto epistemológico y una fundamentación propios: de la razón práctica y de sus condiciones de posibilidad, no desde otros ámbitos de experiencia o de ciencia, provienen los

\footnotetext{
${ }^{35}$ Se cita Schmidt, L: Bioética y Consentimiento Informado. En Revista Latinoamericana de Bioética (Santafé de Bogotá, UMNG) n.10, 2006. pp. 84-135. Íbidem: El problema de los DDHH en el mundo contemporáneo. Memorias del III Congreso Internacional de Ética de la Investigación Científica: La protección de la vida, un compromiso ético, científico y político. Santafé de Bogotá, UMNG, 2006; Íbidem: Derechos de los pacientes y consentimiento informado. Caracas, San Pablo: Colección Biodiké No 7 2009. 64 pp.
} 
conocimientos y los juicios morales, con sus criterios de evidencia y verificación. Así, la actuación de los profesionales de la salud debe estar sustentada en principios humanos fundamentales: la búsqueda de la verdad, el cuidado, el consejo y la solidaridad. 

NOTAS / COMMENTARY 
